\title{
Vitamin D and diacylglycerol reduce fat deposit and change liver lipid metabolism and caecal microflora in rats fed a high-fat diet
}

\author{
Yanan Liu ${ }^{1}$, Baowei Wang ${ }^{1}$, Wenhua $\mathrm{Ge}^{1}$, Mingai Zhang ${ }^{1}$, Min Kong ${ }^{1}$, and Wenlei Fan ${ }^{1}$ \\ ${ }^{1}$ Affiliation not available
}

May 19, 2020

\begin{abstract}
Vitamin D (VD) is associated with the release of leptin, which promotes fat mobilization in the body. Diacylglycerol can reduce triacylglycerol formation. This study evaluated the effects of administration of VD and duck oil diacylglycerol on blood lipids, liver lipid metabolism and gut microflora in rats fed a high-fat diet. A total of 75 rats were allocated randomly to five treatments: non-obesity (NO), obesity control (OC), diacylglycerol:10 mL per kg body weight (DG10), VD:12.5 $\mu$ g per kg body weight (VD12.5), and diacylglycerol + VD: $10 \mathrm{~mL} / \mathrm{d}+12.5 \mu \mathrm{g}$ per $\mathrm{kg}$ body weight (DG10VD12.5). Rats in NO group was fed the diet containing $7 \%$ soybean oil, while the other four groups were fed a high-fat (3.3\% soybean oil and $30.1 \%$ lard). VD and diacylglycerol were orally dosed once per day. The administration of VD+diacylglycerol significantly reduced the body weight gain and fat content. The change of fat deposit was accompanied with the reduction in serum lipids, the increase in the activity of enzymes involved in lipid metabolism in the liver, the suppression of fatty acid synthase and the enhance of carnitine fatty acyl transferase I (CPT1) expression. The administration of VD and diacylglycerol also significant modulated the bacterial diversity profile in the cecum towards that in non-obesity rats. Those results suggest that the administration of VD and diacylglycerol could have significant roles in reducing fat deposit and altering gut microflora in rats fed the high-fat diet.
\end{abstract}

Vitamin D and diacylglycerol reduce fat deposit and change liver lipid metabolism and caecal microflora in rats fed a high-fat diet

Running title: Vitamin D and diacylglycerol reduce fat deposit in rats

Yanan $\mathrm{Liu}^{1}$, Baowei Wang ${ }^{1.2^{*}}$, Wenhua $\mathrm{Ge}^{2}$, Mingai Zhang ${ }^{1}$, Min Kong ${ }^{2}$, and Wenlei Fan ${ }^{1}$

1 Department of Food Science and Engineering, Qingdao Agricultural University, Qingdao 266109, China.

2 National Waterfowl Industry Technical System Nutrition and Feed Function Laboratory, Qingdao 266109, China.

Corresponding author:

Professor Bao-Wei Wang, College of Food Science and Engineering, Qingdao Agricultural University, No. 700 Changcheng Road, Chengyang district, Qingdao 266109, China. E-mail: Wangbw@qau.edu.cn. Phone: $+8613789869968$

Abstract: Vitamin D (VD) is associated with the release of leptin, which promotes fat mobilization in the body. Diacylglycerol can reduce triacylglycerol formation. This study evaluated the effects of administration of VD and duck oil diacylglycerol on blood lipids, liver lipid metabolism and gut microflora in rats fed a highfat diet. A total of 75 rats were allocated randomly to five treatments: non-obesity (NO), obesity control (OC), diacylglycerol:10 mL per $\mathrm{kg}$ body weight (DG10), VD:12.5 $\mu \mathrm{g}$ per $\mathrm{kg}$ body weight (VD12.5), and diacylglycerol + VD: $10 \mathrm{~mL} / \mathrm{d}+12.5 \mu \mathrm{g}$ per $\mathrm{kg}$ body weight (DG10VD12.5). Rats in NO group was fed the 
diet containing $7 \%$ soybean oil, while the other four groups were fed a high-fat (3.3\% soybean oil and $30.1 \%$ lard). VD and diacylglycerol were orally dosed once per day. The administration of VD+diacylglycerol significantly reduced the body weight gain and fat content. The change of fat deposit was accompanied with the reduction in serum lipids, the increase in the activity of enzymes involved in lipid metabolism in the liver, the suppression of fatty acid synthase and the enhance of carnitine fatty acyl transferase I (CPT1) expression. The administration of VD and diacylglycerol also significant modulated the bacterial diversity profile in the cecum towards that in non-obesity rats. Those results suggest that the administration of VD and diacylglycerol could have significant roles in reducing fat deposit and altering gut microflora in rats fed the high-fat diet.

Keywords : Diacylglycerol; Vitamin D; Lipid metabolism; Intestinal microflora; Rats; Obese

\section{Introduction}

With the improvement of living standards, excessive intake of fatty and luscious foods leads to a rapidly increasing trend of obesity, which is a serious public healthy issue all over the world. Obesity can also lead to other diseases, such as inflammation, diabetes, and cardiovascular diseases.

Current therapies for obesity include the use of weight-loss drugs, increasing exercises, and reducing fat intake. Alternative measures are also investigated. Studies have shown that obesity is correlated with vitamin D (VD) deficiency (Leary et al., 2017), thus, supplementation of VD could counteract the symptoms of obesity. For example, the Xi-an Children's Hospital, China conducted a study on VD intervention in 198 overweight and obese children and found that the intervention moderately increased the circulating VD content in obese children, but the response to VD supplementation was weaker than in healthy children (Liu et al., 2008). VD is a natural steroid hormone, which is involved in a variety of physiological functions and can promote the release of leptin, thus promoting mobilization of body fat and reducing insulin resistance (Zhou et al., 2017), but high levels of body fat can lower absorptivity of VD in the gut (Liu et al., 2008). Therefore, obesity people may develop VD deficiency. If so, VD supplementation is necessary in obese people.

Diacylglycerol (DAG) can lower blood lipids, body weight and body fat deposition compared with triglycerides (TG), as well as has other important physiological functions in rats (Diao et al., 2014; Han, 2014). The deposit of body fat depends on dynamic changes in fat synthesis and mobilization, which are regulated by a number of genes. Fatty acid synthase (FAS) is a fatty acid synthetase that catalyzes the generation and elongation of fatty acid chains using acetyl-CoA and malonyl-CoA as the substrates (Menendez et al., 2009). Carnitine acyltransferase I, also known as carnitine palmitoyltransferase I (CPT1), is a key enzyme in fatty acid oxidation. Carnitine can promote fatty acid transfer from cytosol to mitochondria where fatty acid oxidation occurs (Wang et al., 2018). Studies have shown that the carnitine content in liver tissues in high-fat diet induced obese rats decreased significantly compared with healthy rats (Kim et al., 2010), suggesting that the carnitine demand may be increased in obese rats. Wu et al. (2019) found that the expression of CPT1 in the liver tissue of rats on a high-fat was down-regulated while the FAS expression was up-regulated, indicating that fat mobilization could be decreased and fat synthesis increased in high-fat rats.

Aforementioned, VD could increase body fat mobilization via its effect on leptin secretion, and DAG may reduce triglycerides in the body. We, therefore, hypothesized that VD and DAG have synergistic effect, via molecular and microbial mechanisms, on body fat deposit in obese people and test this hypothesis in this study. We used a high fat diet to induce obesity in rats as a model and then applied various DAG and VD treatments. The corresponding changes in cytokines associated with lipids metabolism and the microbiota in the caecum were monitored to assess the effects.

\section{Materials and Methods}

\subsection{Duck oil DAG preparation and detection}

Duck oil DAG was prepared by esterifying glycerol and free fatty acids decomposed from duck oil using an enzymatic method (Wang et al., 2019). Purification of duck oil DAG in the preparation was proceeded using 
molecular distillation equipment (Liu et al., 2020).

The content of DAG was analyzed using a high-performance liquid chromatography method using Agilent 1100 Series (USA) HPLC equipped with a Refractive Index Detector according to the method of Liu et al. (2020).

VD was provided by Shanghai Yuanye Biotechnology Co., Ltd. (CAS:67-97-0, China).

\subsection{Fatty acid composition analysis}

Fatty acid compositions (\% of the total fatty acids) in duck oil DAG preparation and duck oil were analyzed using the gas chromatography method (GB5009-168-2016), and the results are shown in Table 1.

2.3 Animals and experimental design

A total of 75 four-week-old male Sprague-Dawley (SD) rats were used in this study. Rats were housed in stainless steel cages ( 5 rats per cage) in a temperature and humidity controlled room with a 12:12-h light/dark cycle, free access to a commercial diet for rodents and water. The use of the animals and the experimental procedures were approved by the Animal Ethics Committee of the College of Animal Science and Technology, Qingdao Agricultural University, in accordance with the China National Standard - Laboratory Animals Guideline of Welfare and Ethics (2016).

The experiment lasted for 28 days. Rats were acclimated for seven days and fed a commercial diet that contained $7 \%$ soybean oil. At the end of acclimation, rats were allocated into five groups $(\mathrm{n}=15,5$ rats per cage) and randomly assigned to five treatments respectively: non-obesity (NO), obesity control (OC), DAG: $10 \mathrm{~mL}$ per $\mathrm{kg}$ body weight (DG10), VD:12.5 $\mu$ g per kg body weight (VD12.5), and DAG $\times$ VD: $10 \mathrm{~mL} / \mathrm{d} \times$ $12.5 \mu \mathrm{g}$ per $\mathrm{kg}$ body weight (DG10VD12.5). From day 8 to day 28, NO group was continuously fed the diet as in the acclimation period, and the other three groups were fed a high fat (3.3\% soybean oil and $30.1 \%$ lard). Rats in the NO and OC groups was orally drenched once with $10 \mathrm{~mL} / \mathrm{d}$ water, rats in the DAG group drenched with $10 \mathrm{~mL}$ DAG per kg body weight, and rats in the VD group drenched with $12.5 \mu \mathrm{g}$ VD per $\mathrm{kg}$ body weight, and rats in the DAG $\times$ VD group drenched with $10 \mathrm{~mL}$ DAG plus $2.5 \mu \mathrm{g}$ VD per $\mathrm{kg}$ body weight. The drench was administered by oral gavage, once a day. Those treatments lasted for 21 days.

\subsection{Sample collection}

Blood sample (each about $5 \mathrm{~mL}$ ) was taken from rat retro-orbital eye vein into a centrifuge tube, and centrifuged at $2,800 \times \mathrm{g} 4^{\circ} \mathrm{C}$ for $15 \mathrm{~min}$. Serum was harvested, and stored at -40 until analysis.

Once blood sample was taken, rats were immediately dissected, the liver, testicles and visceral fat (around the kidneys, intestinal fat and epididymal fat) were collected, and weighed. The caecum was removed, the content was collected and sampled. All samples were stored at -40 until analysis.

2.5 Body weight and body fat in rats

The weight of rats was weighed weekly using an electronic balance. The perirenal fat, periepididymal fat and mesenteric fat in rats were removed and weighed using an electronic balance. The fat to the body weight percentage was calculated as followings:

The fat ratio $=$ total fat weight $(\mathrm{g}) /$ body weight $(\mathrm{g}) \times 100 \%$

2.6 Serum lipids assays

The concentrations of TG, total cholesterol (TC), high density lipoprotein (HDL), and low density lipoprotein (LDL) in serum were measured using commercial kits (Jiancheng, Nanjing, China) according to the manufacturer's instructions. The absorbance was measured at $450 \mathrm{~nm}$.

2.7 Assays for activity of metabolic enzymes in liver

The sample preparation was briefed as follows: the liver was weighed, cut into tiny pieces in normal saline solution, then homogenized. The homogenate was centrifuged at 500xg at $4 \mathrm{degC}$ for $15 \mathrm{~min}$, the super- 
natant was collected. The aspartate aminotransferase (AST) and alanine transaminase (ALT) activity in the liver was determined using commercial kits (Jiancheng, Nanjing, China) according to the manufacturer's instructions.

\subsection{FAS and CPT 1 mRNA expression in liver}

The liver sample was weighed, cut into tiny pieces in normal saline solution, then homogenized. Total RNA in the liver sample was extracted using a RNAiso Kit (Takara Bio, China) according to the manufacturer's instructions. RNA was reverse-transcribed into cDNA using the 037 A Reverse Transcription Kit (Takara Bio, China). cDNA synthesis conditions were set as follows: $37 \mathrm{degC}$ for $15 \mathrm{~min}, 85 \mathrm{degC}$ for $5 \mathrm{~s}$. PCR amplification was performed in a total volume of $20 \mu \mathrm{L}$, which contained: $2 \mu \mathrm{L}$ cDNA templet, $4 \mu \mathrm{L}$ forward primer $(10 \mu \mathrm{M}), 4 \mu \mathrm{L}$ reverse primer $(10 \mu \mathrm{M}), 10 \mu \mathrm{L}$ SYBR GreenIenzyme-mix, and $7.2 \mu \mathrm{L} \mathrm{ddH}_{2} \mathrm{O}$. Thermal cycling conditions were set as follows: initial denaturation at $95^{\circ} \mathrm{C}$ for $30 \mathrm{~s}$, followed by 40 cycles at $95^{\circ} \mathrm{C}$ for $30 \mathrm{~s}, 95^{\circ} \mathrm{C}$ for $3 \mathrm{~s}$ and $60^{\circ} \mathrm{C}$ for $30 \mathrm{~s}$. Glyceraldehyde 3-phosphate dehydrogenase (GAPDH) was used as internal reference gene. FAS mRNA (forward primer: 5'-TGGACGCCCACCACAAGA3'; reverse primer: 5'-AGAGGAGACAGGTCCAGAGT-3') and CPT1 mRNA (forward primer: 5'CCACAAGTGCCTGTCCGTC-3'; reverse primer: 5'-TCAGGTAGGCTTCGTGGATTC-3') gene expression was detected by SYBR Green I chimeric fluorescence method according to the instructions of SYBR Premix Ex Taq II kit (Takara Bio, China).

\subsection{Microbial diversity in caecum}

Three rats in each group were randomly selected and sacrificed by dispositioning the neck. The caecum was immediated cut off, and the content was collected into a sterile centrifuge tube. Total bacterial DNA in the caecal content sample was extracted using the Power Soil DNA Isolation Kit (MO BIO Laboratories) according to the manufacturer's protocol. DNA quality and quantity were assessed by the ratio of $260 \mathrm{~nm} / 280 \mathrm{~nm}$. Then the extracted DNA was stored at $-80^{\circ} \mathrm{C}$ until the further processes. The V3-V4 region of the bacterial $16 \mathrm{~S}$ rRNA gene was amplified with the common primer pair (forward primer, 5'ACTCCTACGGGAGGCAGCA-3'; reverse primer, 5'-GGACTACHVGGGTWTCTAAT-3') combined with adapter sequences and barcode sequences. PCR amplification was performed in a total volume of $50 \mu \mathrm{L}$, which contained $10 \mu \mathrm{L}$ buffer, $0.2 \mu \mathrm{L}$ Q5 high-fidelity DNA polymerase, $10 \mu \mathrm{L}$ high GC enhancer, $1 \mu \mathrm{L}$ dNTP, $10 \mu \mathrm{M}$ of each primer and $60 \mathrm{ng}$ genome DNA. PCR conditions were set as follows: initial denaturation at $95^{\circ} \mathrm{C}$ for $5 \mathrm{~min}$, followed by 15 cycles at $95^{\circ} \mathrm{C}$ for $30 \mathrm{~s}, 50^{\circ} \mathrm{C}$ for $30 \mathrm{~s}, 72^{\circ} \mathrm{C}$ for $40 \mathrm{~s}$. High-throughput sequencing analysis of bacterial rRNA genes was performed on the purified, pooled sample using the Illumina Hiseq 2500 platform $(2 \times 250$ paired ends $)$ at Biomarker Technologies Corporation, Beijing, China.

\subsection{Statistics analysis}

One-way ANOVA was used for all data analysis. Duncan method was used for multiple comparisons between the means. All analyses were performed using SPSS 17.0 software. P value $<0.05$ was considered as statistically significant.

\section{Results}

\subsection{Effects on body weight and fat}

Fig.1a-d shows the body weight (the initial weight, weight of modeling, and final weight) and body fat percentage in rats. There was no significant difference in the initial body weight between groups (Fig.1a) $(\mathrm{P}>0.05)$. During the treatment period, the body weights of rats in OC, DG, VD12.5, and DG10VD12.5 groups were significantly increased compared with NO group (Fig.1b) $(\mathrm{P}<0.05)$. However, the increment in body weight was lower in VD and DG group $(278.22,280.38, \mathrm{P}<0.05)$, and further lower in DG10VD12.5 group (260.28, $\mathrm{P}<0.05)$ compared with the OC group (298.33) (Fig.1c). For the body fat percentage, rats under DG10D12.5 treatment had the lowest value (4.34) and had lower values under VD and DG treatment (6.03, 5.78) compared with the OC group $(9.04, \mathrm{P}<0.05$ for all).(Fig.1d)

3.2 The effects on serum lipid levels and metabolism enzyme activity 
The effects of DAG and VD on serum lipids are shown in Fig.2a-d. The obesity groups (OC, DG, VD12.5 and DG10VD12.5 treatments) had significant increases in the serum levels of TC, TG, and LDL, whereas reduction in HDL compared with those in NO group $(\mathrm{P}<0.05)$. The DG group or VD group and DG plus VD group had significant influences on the serum lipids compared with those in OC group $(P<0.05)$. For HDL, the highest value was found in rats under the DG10VD12.5 treatment (3.10), and the VD12.5 and DG10 treatment had relatively lower value $(2.84,2.73)$ compared with the OC group (2.02) (Fig.2c). For TG, TC, and LDL, the rats under the DG10VD12.5 treatment had the lowest values $(1.07,3.25$, and 3.16) compared with the OC group (1.81, 4.53, and 4.07) ( $\mathrm{P}<0.05$ for all) (Fig.2a, 2b and 2d).

The effects of DAG and VD on the metabolism enzyme activity in the liver are shown in Fig.3a and 3b. The obesity (OC, DG, VD12.5, and DG10VD12.5 treatments) resulted in significant decreases in the AST and ALT activity compared with those in the NO group $(\mathrm{P}<0.05)$. There were significant differences on the AST and ALT activity between OC and DG10VD12.5 treatments $(\mathrm{P}<0.05)$, however, there were no significant differences between OC and DG10 or VD12.5 group (P > 0.05). For AST, the highest value was found in rats under the DG10VD12.5 treatment (139.14), and the VD12.5 and DG10 treatment had relatively lower value (133.57, 133.81) compared with the OC group (129.42) (Fig.3a). For ALT, the highest value was found in rats under the DG10VD12.5 treatment (49.77), and the VD12.5 and DG10 treatment had relatively lower value $(45.79,46.23)$ compared with the OC group (43.58) $(\mathrm{P}<0.05$ for all) (Fig.3b).

\subsection{The effects on FAS/CPT1 mRNA expression}

The effects of DAG and VD on the expression of genes involved in the PPAR $\gamma / \mathrm{NF}-\varkappa \mathrm{Bp} 65$ pathway are shown in Fig.4a, Fig.4b. Compared with those in the NO group, the obesity significantly lowered the CPT1 value $(\mathrm{P}<0.05)$, but increased the FAS value $(P<0.05)$. There were no significant differences on FAS and CPT1 between NO and DG10VD12.5 treatments $(P<0.05)$. For CPT1 mRNA, the highest value was found in the rats under the DG10VD12.5 treatment (0.90) compared with the OC group (0.46) (Fig.4a). For FAS mRNA, the rats under the DG10VD1.5 treatment had the lower value (1.15) compared with the OC group (1.61) (Fig.4b).

3.4 The correlation between FAS/CPT1 mRNA expression, lipid metabolism, and metabolism enzyme activity

The correlation coefficients between the FAS/CPT1 mRNA expression and the metabolism enzyme activity in the liver, and lipids in serum are listed in Table 2. The FAS mRNA expression was highly and positively correlated with the TC, TG, and LDL concentrations in serum $(\mathrm{P}<0.01)$, but negatively correlated with the ALT and AST activity $(\mathrm{P}<0.01)$. The CPT1 mRNA was highly and negatively correlated with the TC, LDL $(\mathrm{P}<0.01)$, and TG concentrations $(\mathrm{P}<0.05)$, and positively correlated with the ALT and AST activity $(\mathrm{P}<0.01)$.

\subsection{Effects on microbiota in the caecum}

The diversity indices of microbiota, such as operational taxonomic units (OUTs), ACE index, Chao 1 index, Simpson index and Shannon index, in the caecal content are shown in Fig. 5a - 5e. The OC group had significant lower values of the diversity indices compared with those for the other four groups $(\mathrm{P}<0.05)$. For OTUs, the high-fat diet reduced OTUs compared with the NO group, and the DG10VD12.5 treatment (459.33) had highest values compared with the OC group (233.00) (Fig.5a). For ACE index, the high-fat diet reduced ACE index compared with the NO group, and the DG10VD12.5 treatment (421.46), the DG10 treatment (393.81) and the VD12.5 treatment (372.71) had higher values compared with the OC group (263.73) (Fig.5b). For Chao 1 index, the high-fat diet reduced Chao1 index compared with the NO group, and the DG10VD12.5 treatment (423.38) had highest values compared with the OC group (264.79) (Fig.5c). No significant differences in Simpson (Fig.5d) and Shannon (Fig.5e) indices for the microbiota were found between the NO group and the DG10VD12.5 treatment $(\mathrm{P}>0.05)$.

The difference of the microbiota composition was explored using the NMDS analysis. Fig. 6 is the result of the NMDS analysis. The Stress value lesser than 0.2 indicates that the NMDS analysis has certain reliability. 
The distance between the OC group and the NO group was the largest, indicating a significant difference in microbial composition. The distance between DG10VD12.5 treatment and the OC group was closer to that between VD12.5 or DG10 treatment and the OC group.

The microbiota taxonomic distribution at the genus level is shown in Fig. 7a - Fig. 7f. Obesity significantly reduced the abundances of Bacteroides andLactobacillus, whereas increased Allobaculum, Desulfovibrio , Lachnospiraceae, and Alloprevotellagenera compared with those for the NO group $(P<0.05)$. In general, the additions of VD or DAG or VD plus DAG increased the abundances of Bacteroides and Lactobacillus, while reduced the abundances ofLachnospiraceae, Alloprevotella, Desulfovibrio, and Allobaculum . ForLactobacillus, the highest value was found in rats under the VD12.5 treatment (0.159), and the DG10VD12.5 treatment had relatively lower value (0.107) compared with the OC group $(0.023)(\mathrm{P}<0.05$ for all) (Fig.7d). For the other five bacterial genera, the best effects were found in rats under the DG10VD12.5 treatment $(0.051,0.066,0.014,0.034$, and 0.01) compared with the OC group $(0.110,0.013,0.032,0.051$, and 0.066) (Fig.7a, 7b, 7c, 7e and 7f).

The changes in the microbiota composition were also explored using the Line Discriminant Analysis Effect Size (LEfSe) analysis. Fig. 8a and Fig. 8b are the results of the LDA analysis and evolutionary branch diagram. With the LDA threshold set at 4, a total of twenty groups of biomarker with statistical differences were detected. The bacterial taxa with significantly higher abundance in the DG10VD12.5 group wereAcidaminococcaeae and Phascolarctobacterium, while for the DG10 treatment were Rikenellaceae- RC9gut, Collinsella, and Faecalibacterium . In contrast, Campylobacterales ,Helicobacteraceae , Helicobacter , andEpsilonproteobacteria were higher in the OC group, whereasPrevotellaceae -UCG-003, Prevotella -2, Treponema-2,Runminococcaceae-UCG-005, Selenomonadales, Veillonellaceae, Negativicutes, Spirochaetales and Roseburia were higher in the NO group.

\section{Discussion}

We found in this study that the administration of VD or DAG could significantly reduce the body weight gain and the body fat percentage in rats fed a high fat diet and those effect were much stronger when a combination of VD and DAG was applied. The results showed that duck oil DAG and VD may have synergistic effects on reducing body weight gain and fat deposit in obese rats. Murase et al. (2001) showed that body mass and visceral fat were significantly decreased in mice fed a DAG diet compared with a triacylglycerol (TAG) diet, confirming DAG can reduce fat deposit in mice. Guo et al. (2018) found that VD supplementation to obese people could help to reduce the body mass index.

We also found in the present study that duck oil DAG and VD administrations could significantly reduce the circulating lipids, increase AST and ALT enzyme activity in rats fed the high fat diet, and had best effects compare with DAG or VD administration. Ciantti et al. (2007) found that bone marrow stromal cells in the VD receptor gene knockout mice were more likely to differentiate into adipocytes. Other studies have shown that a low serum 25 hydroxyvitamin D concentration is associated with higher blood lipid concentration (Bouillon, 2018), and VD supplementation could reduce blood lipids. Liu et al. (2018) found that the circulating TG level was significantly reduced in response to $1,25(\mathrm{OH})_{2} \mathrm{D}_{3}$ intervention in diabetes rats induced by feeding a high-fat diet. Meng et al. (2004) showed that feeding a high DAG diet significantly reduced the plasma levels of TC, TG, and apolipoprotein b, increased the level of HDL-C, and inhibited the production of neutral fat in rats.

The activities of AST and ALT are important indicators to the liver function (Yan, 2007). He et al. (2016) found that the AST and ALT activity in blood increased in obese rats treated with DAG microcapsule. Piao (2013) found that $\mathrm{VD}_{3}$ had a protective effect on spot necrosis of liver tissue in mice when acute liver injury was induced by administration of carbon tetrachloride. Our results are basically consistent with above results, and also found that duck oil DAG and VD administrations had higher value compare with DAG or VD administration. The results showed that duck oil DAG and VD had the synergistic effects on reducing serum lipids and enhancing AST and ALT activity.

Disorder of lipid metabolism can lead to the development of obesity (Gao, 2011; Jin, 2016; Yang, 2017). 
The principle of promoting fat mobilization should be followed in the treatment of obesity. FAS is a key enzyme to control the synthesis of fatty acids, a process that leads to the accumulation of TG and the risk of hyperlipidemia (Liu, 2013; Lu, 2012). CPT1 is a rate-limiting enzyme for the oxidation of fatty acids (Wang, 2017). In obese people, the increasing level of FAS will inhibit the decomposition of body fat and reduce the activity of CPT1 enzyme, so fat deposit could increase. In this study, we found that duck oil DAG combined with VD and DAG alone or VD alone could significantly down-regulate the FAS expression and up-regulate the CPT 1, and the expression of both genes was highly correlated with the TC, TG, LDL, and HDL concentrations, and the AST and ALT activity, counteracting the fat deposit. Wang et al. (2013) and Xiang et al. (2007) found that DAG decreased the FAS expression and increased the CPT1 expression and opioid melanocortin progenes, thus regulated fatty acid metabolism. Hao et al. (2018) found the AMPdependent protein kinase (AMPK) regulates acetyl-CoA carboxylase (ACC)/CPT1 signaling pathway and promotes fatty acids metabolism and reduce fat accumulation in hypertensive rats. Wu (2012) found that when the expression of lipid metabolism genes in the liver was altered by administration of taurine, the activity of AST and ALT changed. The results of the current experiment are basically consistent with above results. The results showed that duck oil DAG and VD changed the FAS and CPT1 mRNA expression, leading to the corresponding changes in the circulating lipids and the enzymes involved in lipid metabolism in liver, unfavored for fat deposit in obese rats.

Previous reports have provided direct evidence that there is a close relationship between intestinal microflora and the development of obesity. Intestinal microflora disorder can cause chronic and persistent low-grade inflammatory response, lipid metabolism, and intestinal hormone secretion, thus promote the formation of obesity (Cheng et al., 2020). Our results in the present study showed that the obesity significantly changed the composition of the microbiota in the ceacum, and the administration of VD or DAG or a combination of VD and DAG increased Bacteroidales and Lachtobacillus, decreasedLachnospiraceae, Allprevotella, Desulfvibrio, and Allobaculum abundance. Interestingly, Lachnospiraceae and Bacteroidales in the DG10VD12.5 group had higher effects compared with those in the VD12.5 or DG10 group, and Desulfvibrio ,Lactobacillus, Allprevotella, and Allobaculumabundances in the DG10VD12.5 group were no significant difference compared with those in the NO group. It has been suggested that the abundance of Lachtobacillus and Bacteroidales decreases in obesity patients (Doris et al., 2017; Wang et al., 2020) .Lactobacillus exerts a significantly beneficial effect on the gut by inhibiting the growth of pathogenic bacteria, modulating the community of intestinal microflora (Kuugbee et al., 2016; Ou et al., 2011). Desulfovibrionaceae, which is a family of gram-negative sulfate-reducing intestinal bacteria, induces the reduction of sulfate. Researchers have reported that the abundance ofDesulfovibrionaceae is increased in the feces of IBD patients (Berry \& Reinisch, 2013). These results indicate that administration of the DAG and VD could improve the change of microbiota in obese rats.

\section{Conclusion}

The administration of VD plus DAG attenuated the fat deposit and body weight gain induced by feeding a high-fat die in rats. The administration of VD plus DAG improve the expression of FAS and CPT1 genes in the liver of obese mice, promote the activation of fatty acids into fatty acyl CoA and enter the mitochondria for oxidative decomposition, thereby reducing the content of TG and TC in the body and improving liver metabolism.Besides, the change in the microbial diversity by VD and DAG was modulated

towards the profile in the non-obesity group. Through this study, we found that VD combined with DAG has a significant improvement effect on obese rats, therefore, further research is needed to explore the mechanisms on ameliorates obesity by DAG and VD.

\section{Acknowledgements}

This research was funded by Shandong Province Agricultural Application Technology Innovation Project in 2018, and also supported by the Special Fund of National Waterfowl Industry Technology System (No. CARS-43-11) and Graduate Innovation Program Fund of Qingdao Agricultural University (No. QYC201819).

\section{Authorships}


Liu YN, Wang BW, and Ge WH conceived and designed the experiments. Liu YN, Xie YE, and Kong M performed the experiments. Liu YN, Zhang MA, and Fan WL analyzed the data. Liu YN drafted the manuscript. Wang BW and Ge WH reviewed the manuscript. All authors read and approved the manuscript.

\section{Conflict of interest}

The authors have declared no conflict of interest.

\section{References}

Bouillon, R., Marcocci, C., Carmeliet, G., Bikle, D., White, J. H., Dawson-Hughes, B., Lips, P., Munns, C. F., Lazaretti-Castro, M., Giustina, A., Bilezikian, J. (2018). Skeletal and extra-skeletal actions of vitamin D: Current evidence and outstanding questions. Endocrine Reviews, 36 (10): 189-191.

Berry, D., Reinisch, W. (2013). Intestinal microbiota: a source of novel biomarkers in inflammatory bowel diseases?. Best Practice \& Research. Clinical Gastroenterology, 27 (1): 47-58.

Cianferotti, L., Demay, M. B. (2007). VDR-mediated inhibition of DKK1 and SFRP2 suppresses adipogenic differentiation of murine bone marrow stromal cells. Journal of Cellular Biochemistry, 101 (1): 80-8.

Cheng, Y. J., Wang, X. L., Chang, X. Y. (2020). Research progress on the role of intestinal flora in the development of obesity. Jilin Medical Science, 41 (04): 961-964.

Diao, X. Q., Kong, B. H., Chen, Q., Zhao X, X., Liu, Q. (2014). Physiological effects of diacylglycerol and its safety as edible oil. Food industry, 35 (12): 233-236.

Doris, V., Gwen, F., Sara, V. S., Jun, W., Manuela, S., Stephan, T., Kristin V., Jeroen, R. (2017). Prebiotic inulin-type fructans induce specific changes in the human gut microbiota. Gut,66 (11): 1968-1974.

Guo, X. F. (2008). Vitamin D deficiency and childhood obesity. Chinese Journal of Child Health, 26 (12): $1280-1283+1288$.

Gao, L., Kong, X. J., Shi, X. (2011). Effects of electroacupuncture and acupoint cat gut embedding on mRNA expression of lipid metabolism gene PPAR- $\gamma$ mRNA and related lipid metabolism enzymes in simple obese rats. China Acupuncture and Moxibustion, 31 (06): 535-538.

Han, H. N. (2014). Study on the technology and function of preparing goose oil diacylglycerol microcapsule. Qingdao Agricultural University, China.

He, K. L. (2016). Study on technology and function of preparing duck oil diacylglycerol microcapsule catalyzed by immobilized lipase. Qingdao Agricultural University, China.

Hao, M. J., Dang, Y. X., Zhou, X. X., Wang, C. Y. Gao, Y. (2008) Effect of huangqi powder on AMPK/ACC/CPT1 pathway in hyperlipidemia rats. Chinese Journal of Traditional Chinese Medicine, 43 (12): 2586-2592.

Jin, J. Y., Zhang, X., Huo, P. (2016) Relationship between homocysteine level and its metabolic pathway and coronary heart disease in obese adults with simple obesity. Marker Immunoassay and Clinical Studies,23 (11): 1291-1293.

Kim, H. J., Kim, J. H., Noh, S., Hur, H. J., Sung, M. J., Hwang, J. T., Park, J. Ho., Yang, H. J., Kim, M. S., Kwon, D. Young., Yoon, S. H. (2010) Metabolomic analysis of livers and serum from high-fat diet induced obese mice. Journal of Proteome Research, 10 (2): 722-31.

Kuugbee, E. D., Shang, X., Gamallat, Y., Bamba, D., Awadasseid, A., Suliman, M. A., Zang, S., Ma, Y., Chiwala, G., Xin, Y., Shang, D. (2016). Structural change in microbiota by a probiotic cocktail enhances the gut barrier and reduces cancer via TLR2 signaling in a rat model of colon Cancer. Digestive Diseases and Sciences, 61 (10): 2908-2920. 
Leary, P. F., Zamfirova, I., Au, J., McCracken, W. H. (2017). Effect of latitude on vitamin D levels. The Journal of the American Osteopathic Association, 117 (7):433-439.

Liu, R. P., Wu H. B., Sun, H. L., Xiong, F. M. (2008). Effect of vitamin D supplementation on overweight and obese children. China Maternal and Child Health Research, 29 (12): 1566-1569.

Liu, Y. N. Wang, B. W. Xu, H. X. Ge, W. H. Xie, Y. E. Zhang, M. A. Kong, M. Fan, W. L. (2020). Synergistic effect of diacylglycerol and vitamin D in ameliorating dextran sodium sulfate-induced colitis in rats. Lipids, DOI:10.1002/lipd.12248.

Liu, B. B., Yang, Y., Li, Q., Wang H., Wang, L. D. (2008). Effect of 1,25 dihydroxyvitamin D_3 on mRNA expression and triglyceride content of vitamin D receptor, adiposectin receptor 2 and peroxidosomal proliferator-activated receptor in liver of type 2 diabetic rats. Chinese Journal of Diabetes, 26 (01): 55-58.

Liu, Y., Chen, T. T., Fu, L. Y., Ye, Z. N., Li, N. (2013). Effects of lysimachia polysaccharide on the expression of FAS in hyperlipidemia mice. Journal of Jiangxi Agricultural University, 35 (02): 392-397.

Lu, H. Y., Wei, M. Y., Zhang, G., Fu, H. Y. (2012). The relationship between fatty acid synthase and fatty acid metabolism and cytokines. Journal of Cell and Molecular Immunology, 28 (11):1226-1228.

Menendez, J. A., Vazquez-Martin, A.,Ortega, F. J., Fernandez-Real, J. M. (2009). Fatty acid synthase: association with insulin resistance, type 2 diabetes, and cancer. Clinical Chemistry, 55 (3): 425-38.

Murase, T., Mizuno, T., Omachi, T., Onizawa, K., Komine, Y., Kondo, H., Hase, T., Tokimitsu, I. (2001). Dietary diacylglycerol suppresses high fat and high sucrose diet-induced body fat accumulation in C57BL/6J mice. Journal of Lipid Research, 42 : 372-378.

Meng, X. H. (2004). Enzymatic esterification of functional glycerol diester and its function of weight loss. Jiangnan University, China

Ou, C. C., Lin, S. L., Tsai, J. J., Lin, M. Y. (2011). Heat-killed lactic acid bacteria enhance immunomodulatory potential by skewing the immune response toward Th1 polarization. Journal of Food Science,76 (5): M260-7.

Pu, M. L. (2013) Protective effect of vitamin D_3 on acute liver injury induced by carbon tetrachloride in mice. Yanbian University, China.

Wang, L. L., Qi, X., Li, J. L. (2008). Progress of metabolomics in obesity. Chemistry of Life, 38 (06): 803-808.

Wu, Y. X., Li, Y. X., Ye, G. Y., Mao, R. J., Zhang, J. (2019). Effect of Hawthorn fat burner capsule on lipid metabolism in obese mice induced by diet. Pharmacy Today, 07 (29): 449-452+460.

Wang, B. W., Zhang, M. A., Ge, W. H., He, K. L., Cheng, F. S. (2019). Microencapsulated duck oil diacylglycerol: Preparation and application as anti-obesity agent. LWT-Food Science and Technology, DOI:10.1016/j.lwt.2018.11.061.

Wang, R., Su, D. W., Pang, Z. J., Zhou X. Y., Gong, B. S., He, X. B., Yao, Z. Z. (2017). Research progress on the relationship between carnitine palmidil transferase 1c and tumor. Chemistry of Life,37 (02):207-211.

Wang, F. (2013). Effect of 1, 3-glycerodiester cream fat on fat metabolism in SD rats and its mechanism. Anhui Agricultural University, China.

Wu, G. F. (2012). Prevention and treatment of alcoholic fatty liver in rats with taurine and its mechanism. Shenyang Agricultural University, China.

Wang, C., Chao, H. P., Xie Y. D. (2020). Analysis of the relationship between the effect of inulin on weight loss in obese mice induced by high-fat and high-sugar diet and its regulation of intestinal flora. Journal of Anhui Medicine, 24 (04): 666-670. 
Xiang, S. S. (2007). Effects of glycerol diester on lipid metabolism in SD rats and its mechanism. Fourth Military Medical University, China.

Yan, Q., Xie, S., Zhu, X., Lei, W., Yang, Y. (2007) Dietary methionine requirement for juvenile rockfish, Sebastes schlegeli. Aquaculture Nutrition, 13 (3): 163-169.

Yang, H. L., Mao, L. L., Ran, J. R., Tan, M.G. (2017). Investigation of serum HCY level in 200 obese Tujia children. Journal of Clinical Laboratory, 6 (02): 153-155.

Zhou, H. X., Xu, Q., Cai, H. S. (2017). Effect of high dose vitamin D on prevention and treatment of obese pregnant women with gestational diabetes. Chinese General Practice, 15 (11): 1911-1914.

Table 1 Fatty acids compositions (g/100 g fatty acids) of duck oil diacylglycerol preparation and duck oil

\begin{tabular}{lll}
\hline Fatty acids & Duck oil diacylglycerol & Duck oil \\
$16: 0$ & 19.17 & 26.13 \\
$16: 1$ & 6.53 & 6.01 \\
$18: 0$ & 5.83 & 18.12 \\
$18: 1$ & 45.69 & 38.73 \\
$18: 2$ & 20.65 & 9.04 \\
$18: 3$ & 1.54 & - \\
$20: 0$ & 0.31 & 0.21 \\
$20: 1$ & - & 1.52 \\
$22: 0$ & 0.27 & 0.24 \\
\hline
\end{tabular}

Table 2 The correlation coefficients between FAS 、 CPT1 mRNA expression, blood lipids, and enzyme activity in liver of rats

\begin{tabular}{lll}
\hline Items & FAS mRNA & CPT1 mRNA \\
TC & $0.817^{* *}$ & $-0.893^{* *}$ \\
TG & $0.757^{* *}$ & $-0.634^{*}$ \\
HDL & -0.381 & 0.460 \\
LDL & $0.805^{* *}$ & $-0.842^{* *}$ \\
ALT & $-0.827^{* *}$ & $0.862^{* *}$ \\
AST & $-0.847^{* *}$ & $0.893^{* *}$ \\
\hline
\end{tabular}

*significant $(P ; 0.05)$. **Highly significant $(P ; 0.01)$.

Fig.1a Effects of duck oil diacylglycerol (DAG) and vitamin D (VD) on initial weight in rats. NO: nonobesity; OC: obesity control; DG10 refers to DAG dose at $10 \mathrm{~mL} / \mathrm{kg}$ BW per day; VD12.5 stands for VD dose at $12.5 \mu \mathrm{g} / \mathrm{kg}$ BW per day.

Fig.1b Effects of duck oil diacylglycerol (DAG) and vitamin D (VD) on model weight in rats. NO: nonobesity; OC: obesity control; DG10 refers to DAG dose at $10 \mathrm{~mL} / \mathrm{kg}$ BW per day; VD12.5 stands for VD dose at $12.5 \mu \mathrm{g} / \mathrm{kg}$ BW per day.

Fig.1c Effects of duck oil diacylglycerol (DAG) and vitamin D (VD) on final weight in rats. NO: non-obesity; OC: obesity control; DG10 refers to DAG dose at $10 \mathrm{~mL} / \mathrm{kg}$ BW per day; VD12.5 stands for VD dose at $12.5 \mu \mathrm{g} / \mathrm{kg}$ BW per day.

Fig.1d Effects of duck oil diacylglycerol (DAG) and vitamin D (VD) on body fat percentage in rats. NO: non-obesity; OC: obesity control; DG10 refers to DAG dose at $10 \mathrm{~mL} / \mathrm{kg}$ BW per day; VD12.5 stands for VD dose at $12.5 \mu \mathrm{g} / \mathrm{kg}$ BW per day. 
Fig.2a Effects of duck oil diacylglycerol (DAG) and vitamin D (VD) on serum TG in obese rats. TG: triacylglycerol. NO: non-obesity; OC: obesity control; DG10 refers to DAG dose at $10 \mathrm{~mL} / \mathrm{kg}$ BW per day; VD12.5 stands for VD dose at $12.5 \mu \mathrm{g} / \mathrm{kg}$ BW per day.

Fig.2b Effects of duck oil diacylglycerol (DAG) and vitamin D (VD) on serum TC. TC: total cholesterol in obese rats. NO: non-obesity; OC: obesity control; DG10 refers to DAG dose at $10 \mathrm{~mL} / \mathrm{kg}$ BW per day; VD12.5 stands for VD dose at $12.5 \mu \mathrm{g} / \mathrm{kg}$ BW per day.

Fig.2c Effects of duck oil diacylglycerol (DAG) and vitamin D (VD) on serum HDL in obese rats. HDL: high density lipoprotein. NO: non-obesity; OC: obesity control; DG10 refers to DAG dose at $10 \mathrm{~mL} / \mathrm{kg}$ BW per day; VD12.5 stands for VD dose at $12.5 \mu \mathrm{g} / \mathrm{kg}$ BW per day.

Fig.2d Effects of duck oil diacylglycerol (DAG) and vitamin D (VD) on serum LDL in obese rats. LDL: low density lipoprotein. NO: non-obesity; OC: obesity control; DG10 refers to DAG dose at $10 \mathrm{~mL} / \mathrm{kg} \mathrm{BW}$ per day; VD12.5 stands for VD dose at $12.5 \mu \mathrm{g} / \mathrm{kg}$ BW per day.

Fig.3a Effects of duck oil diacylglycerol (DAG) and vitamin D (VD) on AST in obese rats. AST: aspartate aminotransferase. NO: non-obesity; OC: obesity control; DG10 refers to DAG dose at $10 \mathrm{~mL} / \mathrm{kg}$ BW per day; VD12.5 stands for VD dose at $12.5 \mu \mathrm{g} / \mathrm{kg}$ BW per day.

Fig.3b Effects of duck oil diacylglycerol (DAG) and vitamin D (VD) on ALT in obese rats. ALT: alanine transaminase. NO: non-obesity; OC: obesity control; DG10 refers to DAG dose at $10 \mathrm{~mL} / \mathrm{kg}$ BW per day; VD12.5 stands for VD dose at $12.5 \mu \mathrm{g} / \mathrm{kg}$ BW per day

Fig.4a Effects of duck oil diacylglycerol (DAG) and vitamin D (VD) on FAS mRNA expression in liver of obese rats. NO: non-obesity; OC: obesity control; DG10 refers to DG dose at $10 \mathrm{~mL} / \mathrm{kg}$ BW per day; VD12.5 stands for VD dose at $12.5 \mu \mathrm{g} / \mathrm{kg}$ BW per day.

Fig.4b Effects of duck oil diacylglycerol (DAG) and vitamin D (VD) on CPT1 mRNA expression in liver of obese rats. NO: non-obesity; OC: obesity control; DG10 refers to DAG dose at $10 \mathrm{~mL} / \mathrm{kg}$ BW per day; VD12.5 stands for VD dose at $12.5 \mu \mathrm{g} / \mathrm{kg}$ BW per day.

Fig.5a Effects of duck oil diacylglycerol (DAG) and vitamin D (VD) on OTUs. OTUs: operational taxonomic units. NO: non-obesity; OC: obesity control; DG10 refers to DAG dose at $10 \mathrm{~mL} / \mathrm{kg} \mathrm{BW}$ per day; VD12.5 stands for VD dose at $12.5 \mu \mathrm{g} / \mathrm{kg}$ BW per day.

Fig.5b Effects of duck oil diacylglycerol (DAG) and vitamin D (VD) on ACE index. NO: non-obesity; OC: obesity control; DG10 refers to DAG dose at $10 \mathrm{~mL} / \mathrm{kg}$ BW per day; VD12.5 stands for VD dose at 12.5 $\mu \mathrm{g} / \mathrm{kg} \mathrm{BW}$ per day.

Fig.5c Effects of duck oil diacylglycerol (DAG) and vitamin D (VD) on Chao1 index. NO: non-obesity; OC: obesity control; DG10 refers to DAG dose at $10 \mathrm{~mL} / \mathrm{kg}$ BW per day; VD12.5 stands for VD dose at 12.5 $\mu \mathrm{g} / \mathrm{kg}$ BW per day.

Fig.5d Effects of duck oil diacylglycerol (DAG) and vitamin D (VD) on Simpson index. NO: non-obesity; OC: obesity control; DG10 refers to DAG dose at $10 \mathrm{~mL} / \mathrm{kg}$ BW per day; VD12.5 stands for VD dose at $12.5 \mu \mathrm{g} / \mathrm{kg}$ BW per day.

Fig.5e Effects of duck oil diacylglycerol (DAG) and vitamin D (VD) on Shannon index. NO: non-obesity; OC: obesity control; DG10 refers to DAG dose at $10 \mathrm{~mL} / \mathrm{kg}$ BW per day; VD12.5 stands for VD dose at $12.5 \mu \mathrm{g} / \mathrm{kg}$ BW per day.

Fig.6 The NMDS analysis on the cecal content. cecumM: OC group, cecumD: NO group, cecum3: DG10 group, cecum4: VD12.5 group, cecum5: DG10VD12.5 group. NO: non-obesity, OC: obesity control, DG 10 refer to diacylglycerol doses at $10 \mathrm{~mL} / \mathrm{kg}$ BW per day, VD 12.5 stand for vitamin D doses at $12.5 \mu \mathrm{g} / \mathrm{kg}$ BW per day. 
Fig.7a Distribution of Allbaculum in the caecal content. NO: non-obesity; OC: obesity control; DG10 refers to diacylglycerol dose at $10 \mathrm{~mL} / \mathrm{kg} \mathrm{BW}$ per day; VD12.5 stands for Vitamin D dose at $12.5 \mu \mathrm{g} / \mathrm{kg}$ BW per day.

Fig.7b Distributions of Bacteroidales in the caecal content. NO: non-obesity; OC: obesity control; DG10 refers to diacylglycerol dose at $10 \mathrm{~mL} / \mathrm{kg}$ BW per day; VD12.5 stands for Vitamin D dose at $12.5 \mu \mathrm{g} / \mathrm{kg}$ BW per day.

Fig.7c Distributions of Lachnospiraceae in the caecal content. NO: non-obesity; OC: obesity control; DG10 refers to diacylglycerol dose at $10 \mathrm{~mL} / \mathrm{kg}$ BW per day; VD12.5 stands for Vitamin D dose at $12.5 \mu \mathrm{g} / \mathrm{kg}$ BW per day.

Fig.7d Distributions of Lactobacillus in the caecal content. NO: non-obesity; OC: obesity control; DG10 refers to diacylglycerol dose at $10 \mathrm{~mL} / \mathrm{kg}$ BW per day; VD12.5 stands for Vitamin D dose at $12.5 \mu \mathrm{g} / \mathrm{kg}$ BW per day.

Fig.7e Distributions of Desulfovibrio in the caecal content. NO: non-obesity; OC: obesity control; DG10 refers to diacylglycerol dose at $10 \mathrm{~mL} / \mathrm{kg}$ BW per day; VD12.5 stands for Vitamin D dose at $12.5 \mu \mathrm{g} / \mathrm{kg}$ BW per day.

Fig.7f Distributions of Alloprevotella in the caecal content. NO: non-obesity; OC: obesity control; DG10 refers to diacylglycerol dose at $10 \mathrm{~mL} / \mathrm{kg}$ BW per day; VD12.5 stands for Vitamin D dose at $12.5 \mu \mathrm{g} / \mathrm{kg}$ BW per day.

Fig.8a The LDA analysis diagram on the cecal content. Group1: NO group; Group2: OC group; Group3: DG10 group; Group5: DG10VD12.5 group. NO: non-obesity; OC: obesity control; DG10 refers to diacylglycerol dose at $10 \mathrm{~mL} / \mathrm{kg}$ BW per day; VD12.5 stands for Vitamin D dose at $12.5 \mu \mathrm{g} / \mathrm{kg}$ BW per day.

Fig.8b The evolutionary branch diagram on the caecal content. Group1: NO group; Group2: OC group; Group3: DG10 group; Group5: DG10VD12.5 group. NO: non-obesity; OC: obesity control; DG10 refers to diacylglycerol dose at $10 \mathrm{~mL} / \mathrm{kg}$ BW per day; VD12.5 stands for Vitamin D dose at $12.5 \mu \mathrm{g} / \mathrm{kg}$ BW per day.

\section{Hosted file}

Figures.docx available at https://authorea.com/users/324195/articles/452460-vitamin-dand-diacylglycerol-reduce-fat-deposit-and-change-liver-lipid-metabolism-and-caecalmicroflora-in-rats-fed-a-high-fat-diet 